\title{
Keeping astronomers in the dark
}

\author{
Making sure the night sky stays dark near astronomical observatories makes good sense. For \\ radioastronomers the problem is more difficult, but the solution is the same.
}

\section{Tucson, Arizona}

ANYONE who lives in a modern city or its suburbs will be quite mystified by the multitude of books on amateur astronomy meant to guide the novice around the spectacular sights of the night sky. The brightest stars in the best known constellations may be visible, but the Pleiades will be little more than a blur, while the Orion nebula and even the Milky Way might as well be fabrications. To such a city-dweller, the night sky seen from a dark and remote spot can be a breathtaking surprise, and may cause a sudden understanding of why some people devote their lives to observing the stars.

But there is more than social psychology to worry about. Astronomers at the big observatories are everywhere finding that city lights are creeping up on them, restricting the area of sky that can be usefully observed to the higher altitudes, and making it more difficult for instruments to pluck faint objects out of the background. The Mount Wilson Observatory, in the mountains near Los Angeles, has already been made useless; the night sky there is artificially five or ten times brighter than the natural sky, and this 'light pollution' is aggravated by real pollution, for dust and foreign gases also scatter and absorb light.

Lately, astronomers have been retreating to higher and more distant mountain tops, both to escape civilization and to get above as much of the atmosphere as possible. Three of the most important modern observatories are at Cerro Tololo in the Chilean Andes, on Mauna Kea in Hawaii and on La Palma in the Canary Islands. But even with continuing expansion, these sites cannot accommodate the world's demand for observing time, and efforts to forestall and reverse the deterioration of observing conditions at other observatories are essential.

At the Kitt Peak National Observatory, about 50 miles from Tucson, Arizona, there is some success to report. Since 1972, the city of Tucson has enacted a number of local ordinances governing the type of street lighting to be used, and the brightness and pattern of lights for store displays and advertising, which require some lights to be turned off at night. Astronomers have been astute in allying their good cause with others, such as energy conservation, and have found the electric utility companies co-operative in recommending higher-efficiency shielded lighting to their customers.

Some of the changes seem absurdly simple. Installing lights at the top of a billboard pointing down rather than at the bottom pointing up makes a huge difference, as does a newer regulation requiring the background of advertising posters to be darker than the lettering. Local ordinances mean advertisers and shopowners must restrain their natural extravagance, but if the rules are consistently applied nobody seems to object.

The result of this cooperation between the astronomers and the city officials is that artificial light at present makes up only about 8 per cent of the night-sky brightness at Kitt Peak. And some of that comes from Phoenix - 100 miles away, but a bigger city than Tucson - which has also recently enacted light-control measures. So while astronomers are pleased with their successes, they are fighting a growing trend, especially as Arizona is working to attract new industries and a younger population.

Big astronomy has come more recently to Mauna Kea, and scientists there have been able to work with the island government as the observatory has grown. In some ways their task has been easier than at Kitt Peak: energy conservation is already a public concern on Hawaii, and the observatory, having provided jobs and money to an economy which depends largely on tourism, is regarded as an important asset to the island.

That optical astronomy is a visible, benign science which generally enjoys good public relations has contributed to the success of light-control programmes. Radioastronomers are not so lucky. Their science is more mysterious to the layman, and radio interference can reach them from around the world. Any attempts by scientists in the United States to protect and regulate certain parts of the radio spectrum must work their way upwards through the National Science Foundation (NSF) to the Federal Communications Commission (FCC) and the National Telecommunications and Information Administration (NTIA), and thence into the international arena.

Radioastronomers cannot directly guard their own interests, and the FCC and NTIA are said to be less sympathetic to scientists than to the communications industry, which regards vacant parts of the electromagnetic spectrum as the mining industry regards the national parks.
Radioastronomers would like to maintain clear spots roughly in every frequency decade of the spectrum, along with a few particular reservations for important radio lines (mostly of hydrogen). But last autumn a World Administrative Radio Conference made new allocations, principally for users of mobile radio equipment, of frequencies near $1.6 \mathrm{GHz}$, and recommended a complete review of all allocations in the $1-12 \mathrm{GHz}$ range.

NSF, which represents the astronomical community in international dealings, is gearing up to defend itself not only against the global communications industry but also against the suspicions of some other countries that the protection of clear bands is really being promoted on behalf of the owners of spy satellites.

Although global negotiations on the use of the radio spectrum are beyond the direct reach of astronomers, some local concerns have been dealt with more directly and with consequent success. The radio telescopes at Green Bank, West Virginia, have been protected by the establishment of a 13,000 square mile Radio Quiet Zone, within which radio operations are strictly controlled. At the Very Large Array, near Socorro, New Mexico, there is no comparable formal protection, but the site is remote and the few neighbours (the White Sands Missile Range and the US Forest Service) have been friendly.

Technical ingenuity can also overcome some conflicts. It is possible for radioastronomers to share a frequency with a local user who agrees to switch his equipment off for perhaps the first 200 milliseconds of every second. Interferometric observations using several telescopes in parallel are less vulnerable to local interference because the methods of data analysis tend to remove small-scale disturbances.

Terrestrial pollution, physical and electromagnetic, threatens astronomy of all kinds. An optimistic answer is to put astronomy in space, but even with the Hubble telescope soon to be in orbit and plans for space-based radiotelescopes widely discussed, no more than a tiny fraction of the observations that need to be done will be made from outside the atmosphere. But astronomers can achieve a great deal through their own efforts, and they are fortunate to be defending a science which has natural popular appeal and which can be explained in simple terms with relative ease. David Lindley 